\title{
FILOSOFÍA Y EXPERIENCIA EN HEGEL: LA FENOMENOLOGÍA DEL ESPÍRITU Y LA ENCICLOPEDIA DE LAS CIENCIAS FILOSÓFICAS EN DIÁLOGO
}

\author{
Fernando Forero \\ doi: 10.11144/Javeriana.uph37-75.fehf
}

\section{RESUMEN}

En la versión del sistema de la Enciclopedia de las ciencias filosóficas, el propio Hegel establece un entronque entre la Fenomenología del espiritu y su filosofía posterior. Este artículo examina una vez más esta conexión. Si uno de los aspectos característicos de la Fenomenología consiste en que ella no se identifica plenamente con la reflexión filosófica sistemática ni representa un puro tratado sobre la experiencia humana, sino que se elabora a horcajadas entre estos dos extremos, lo que se hace evidente en la "Fenomenología" de la Enciclopedia es que allí la obra parece alejarse de la exposición del camino de la experiencia de la conciencia natural. Esto obedece a que la "Fenomenología" de la Enciclopedia ya no parece establecer una conexión tan clara entre filosofía y experiencia o, en otra formulación, no parece asumir que los planteamientos filosóficos ganen su verdad mediante su encarnación en la praxis humana. Al hilo de la pregunta por la relación entre la Fenomenología del espiritu y la Enciclopedia de las ciencias filosóficas, este artículo considera la idea de filosofía presente en ambas obras, así como la enigmática relación entre experiencia y filosofía.

Palabras clave: Hegel; filosofía; experiencia; sistema; ciencia

Universidad Nacional de Colombia, Bogotá, Colombia.

Correo electrónico: jofforeropi@unal.edu.co

Para citar este artículo: Forero, F. (2019). Filosofía y experiencia en Hegel: la Fenomenología del espíritu y la Enciclopedia de las ciencias filosóficas en diálogo. Universitas Philosophica, 37(75), 17-43. ISSN 0120-5323, ISSN en línea 2346-2426. doi: 10.11144/Javeriana.uph37-75.fehf 


\title{
PHILOSOPHY AND EXPERIENCE IN HEGEL: THE PHENOMENOLOGY OF THE SPIRIT AND THE ENCYCLOPEDIA OF PHILOSOPHICAL SCIENCES IN DIALOGUE
}

\begin{abstract}
In the Encyclopedia of Philosophical Sciences Hegel himself establishes a relationship between the Phenomenology of the Spirit and the development of his subsequent philosophical thought. This article reexamines this connection. If one of the characteristic aspects of the Phenomenology is that it is neither fully an expression of systematic philosophical reflection, nor does it represent a pure analysis of human experience, but is elaborated between these two extremes, what is evident in the Encyclopedia's "Phenomenology" is also that in it the work seems to move away from the exposition of the path of the experience of natural consciousness. This is because the Encyclopedia's "Phenomenology", no longer seems to establish a connection between philosophy and experience or, in other words, it does not assume that philosophical approaches gain their truth through their incarnation in human praxis. Following the question about the relationship between the Phenomenology of the Spirit and the Encyclopedia of Philosophical Sciences, this article considers the idea of philosophy present in both works, as well as the enigmatic relationship between experience and philosophy.
\end{abstract}

Keywords: Hegel; philosophy; experience; system; science 
¿EN QUÉ CONSISTE EL PROYeCto de elaborar una Enciclopedia de las ciencias filosóficas? La Enciclopedia es una obra que Hegel reelaboró varias veces y que constituye su exposición definitiva del sistema. Se trata de la presentación sistemática de cómo la realidad en todos sus órdenes, niveles y esferas está determinada por una misma estructura racional, a la que Hegel nombra de diferentes maneras y con distintos acentos: Absoluto, Concepto, Idea. La Enciclopedia es la exposición total del proceso del Absoluto, esto es, del sistema hegeliano. La obra se compone de tres secciones. La primera de ellas es la "Ciencia de la lógica”, que presenta la conceptualidad de la Idea, pero antes de que entre en la realidad (Hegel, 2016a, pp. 367 y ss.). La Lógica expone la idea como una red de determinaciones conceptuales en su pureza; se intenta captar allí las relaciones racionales en su forma interna, es decir, sin referirlas aún a procesos reales. Se trata de la exposición de la racionalidad presente en la realidad, pero antes de hacerse efectiva o, dicho en términos más hegelianos, es "la idea en su inmediatez" (Hegel, 2016a, p. 388). El sistema estaría entonces incompleto si se quedara a este nivel lógico. Tiene que mostrar cómo esos conceptos se presentan en la realidad y por eso la exposición transita a las otras dos partes del sistema, a saber, la "Filosofía de la naturaleza" y la "Filosofía del espíritu". La Lógica es la razón en su conceptualidad pura, de la que luego debemos determinar cómo ingresa y puebla el mundo natural en la "Filosofía de la naturaleza" y reconocer en qué sentido configura el mundo humano en la "Filosofía del espíritu". La segunda parte del sistema es entonces la "Filosofía de la naturaleza", que describe la manera como las determinaciones conceptuales de la forma lógica organizan y atraviesan el mundo natural. Al presentarse la forma lógica en la realidad natural, sus determinaciones se vinculan unas a otras a través de la lógica de la negatividad; en otras palabras, la "Filosofía de la naturaleza" es la exposición dialéctica de los conceptos con que se piensa y determina la naturaleza, y su propósito es el de presentar en sus relaciones "la forma absoluta en la realidad natural" (Hegel, 2016g, p. 11). Allí se expone la idea en su exterioridad. La última parte del sistema es la "Filosofía del espíritu". Hegel (2016h, p. 9) afirma que allí la idea se reunifica, se conoce o reconoce a sí misma progresivamente. También esta "Filosofía del espíritu” está en el marco de una Enciclopedia de las ciencias filosóficas, es decir, la del espíritu es también una ciencia de la que se ocupa la filosofía, de manera que es el modo como advertimos que la racionalidad o la idea articula y 
permea el mundo humano. En la esfera del espíritu alcanzamos una racionalidad que se sabe a sí misma y por eso Hegel (2016h, p. 11) afirma que, si el rasgo de la naturaleza es la exterioridad, en el sentido de que no sabe de sí, el del espíritu es la "interioridad", pues constituye el proceso por el cual nos hacemos conscientes de la racionalidad que nos atraviesa.

La Enciclopedia es, entonces, la gran presentación del sistema. Sabemos que para gran parte de las secciones de las que se compone esta obra Hegel ofreció lecciones aparte que después fueron compendiadas, y que elaboraban un apartado del sistema y profundizaban en él. Así, hay lecciones sobre filosofía de la naturaleza, antropología, filosofía de la historia, arte, religión, historia de la filosofía, etc. De tales ampliaciones del sistema, las únicas que Hegel preparó para la publicación fueron la Filosofía del derecho y, antes de la exposición definitiva del sistema, la Ciencia de la lógica. Tenemos entonces en la obra del Hegel maduro, por un lado, el sistema presentado en la Enciclopedia de las ciencias filosóficas y, por otro, las Lecciones derivadas de sus cursos y los textos en los que él indagaba a fondo y precisaba las partes del sistema aisladamente. El asunto es que hay una sección de la Enciclopedia denominada "Fenomenología del espíritu" (Hegel, 2016h, pp. 199 y ss.). Esta sección plantea un interrogante clásico, a saber, si la Fenomenología del espiritu de 1807 constituye a su vez una ampliación o elaboración sistemática de la "Fenomenología del espíritu" de la Enciclopedia, o si más bien esta obra no hace parte del sistema, de manera que ella queda por fuera del proyecto filosófico del Hegel maduro. En efecto, una de las cuestiones más espinosas que ha acompañado toda la recepción de la Fenomenología de 1807 es la que concierne a su lugar dentro del sistema general que Hegel desarrolla posteriormente. Ciertamente, en el mapa global del sistema que ofrece la Enciclopedia de las ciencias filosóficas hay una sección titulada "Fenomenología del espíritu"; pero se trata tan solo de un esquema estrecho de la obra de 1807, que únicamente toma en cuenta las figuras que van desde el capítulo titulado "Conciencia" hasta el que lleva por nombre "Razón", dejando sin considerar las figuras del "Espíritu” y demás. Para una mirada que solo se atenga al índice de las obras en cuestión, salta a la vista que aquello que aparece dentro de la sección "Espíritu subjetivo" como "Fenomenología del espíritu" no es lo mismo que Hegel expuso como $\mathrm{Fe}$ nomenología del espiritu en 1807. Pues bien, este artículo vuelve sobre este debate clásico que no deja de animar a los especialistas. Nos preguntamos por el lugar 
que ocupa el gran texto de 1807 al interior del sistema del Hegel maduro. Podemos elaborar este célebre asunto empezando por situar a la "Fenomenología del espíritu" dentro del proyecto de una filosofía del espíritu, tal y como se presenta en la Enciclopedia de las ciencias filosóficas. Una vez presentado lo que elabora la "Fenomenología" de la Enciclopedia, podremos volver a la Fenomenología del espiritu de 1807 para destacar su particularidad.

\section{El lugar de la Fenomenología del espíritu al interior de la Enciclopedia de las ciencias filosóficas}

LA FILOSOFÍA DEL ESPÍRITU no es sino la actividad de manifestarse de la racionalidad de la Idea en el ámbito de lo humano, es decir, del comparecer para nosotros una racionalidad presente en nuestro mundo concreto; se trata de la Idea haciéndose efectiva, mostrándose, revelándose a sí misma en el ámbito de las producciones propiamente humanas. En el parágrafo 384 de la Enciclopedia Hegel (2016h, p. 29) habla del manifestarse de la racionalidad de la idea en el espíritu como un devenir que no es ciego para sí mismo, sino más bien el hacer propio un mundo que parece externo. El espíritu pone lo exterior como algo propio o como mundo suyo; hacemos de la realidad algo que introducimos en espacios de sentido y nos familiarizamos con ello, de modo que debemos entender el espíritu como el manifestarse de una racionalidad que implica recoger e interiorizar en unidades de sentido cada vez más profundas lo que parece externo, separado entre sí y suelto, o, en otra formulación, como un acoger e interiorizar lo que comparece como ajeno.

En el parágrafo 385 de la Enciclopedia Hegel sugiere que podemos estudiar esa actividad espiritual únicamente desde el punto de vista de la subjetividad, en cuyo caso estamos en el ámbito del "Espíritu subjetivo". Allí se consideran las capacidades humanas que nos permiten hacer ese proceso de interiorización de lo presuntamente externo. El del "Espíritu subjetivo" es un análisis de la posibilidad humana de apropiarse o traer el mundo a nuestra realidad de sentido solo desde un examen de nuestras capacidades. La segunda parte de la "Filosofía del espíritu” es el "Espíritu objetivo" y considera la cuestión de cómo esa inteligibilidad racional termina condicionando toda la realidad objetiva del mundo humano; se trata de ver cómo el ser humano se apropia de su mundo objetivo y 
termina cobijando ese mundo presuntamente externo y extraño. Allí Hegel estudia las formas básicas de organización humana del mundo; se examinan, pues, el derecho, la moral, las ideas de lo bueno, lo justo, instituciones como la familia o constructos como la sociedad y el Estado, que son, en el fondo, las formas primarias en que se organizan y estructuran las comunidades. Con ello, se evidencia que la racionalidad de la Idea no solo atraviesa al ser humano individual, sino también la realidad social en que vivimos. La última parte de la "Filosofía del espíritu" corresponde al "Espíritu absoluto", en la que ya no se observa al espíritu en su lado subjetivo ni objetivo, pues se reconoce una conceptualidad general que lo agrupa todo. Vemos entonces la Idea, primero desde el lado de la conciencia, después desde el lado del mundo y al final desde lo absoluto, como una racionalidad que abarca los dos momentos anteriores. Las formas de ver esa racionalidad que reúnen al individuo con su mundo son el arte, la religión y la filosofía (Hegel, 2016h, p. 32).

La "Fenomenología del espíritu" aparece en la segunda sección del "Espíritu subjetivo", es decir, de la sección que se propone realizar un análisis de las posibilidades en que está todo individuo de inmiscuirse en una totalidad racional. Dado que el "Espíritu subjetivo" considera la manera como la racionalidad que Hegel llama Idea determina al individuo, se aísla allí al sujeto para estudiarlo en sus funciones antropológicas, racionales y psicológicas. Lo que interesa es ver al individuo permeado por una racionalidad superior que, por así decir, determina también sus capacidades subjetivas. Hegel distingue en esta sección entre el alma, de la que se ocupa la "Antropología", considerando al individuo en su inmediatez o ligado estrechamente a las determinaciones que lo anclan a la naturaleza; la conciencia, que examina la "Fenomenología del espíritu" y que tiene que ver con el proceso por el cual una consciencia se va confrontando con su objeto; y el espiritu, que es determinado en la "Psicología" y que presenta al espíritu individual que se sabe en una totalidad sustancial.

Pues bien, Hegel dice en la Enciclopedia que la "Fenomenología del espíritu" nos lleva al terreno de la razón y a superar las limitaciones del ámbito del entendimiento. ¿En qué consiste este tránsito? Podemos comprender esta dialéctica acudiendo a la diferencia entre razón y entendimiento en Kant. Sabemos que para Kant el entendimiento está limitado a lo fenoménico, mientras que la razón tiene una tendencia a ir más allá de lo puramente sensible, aspira a lo infinito, solo 
que al avanzar hacia allá entra en contradicciones. El argumento de Hegel tiene alguna similitud con el planteamiento de Kant. Su idea es que la razón tiene la vocación de unificar. Mientras que el entendimiento está limitado a un mundo puramente fenoménico u objetivo, esto es, aún confronta al sujeto con su realidad, la razón tiene una vocación a ir más allá de esa confrontación o, en otros términos, a unificar el sujeto con su mundo. En Kant la razón aún se queda en la ruptura y la escisión, mientras que en Hegel el poder de la razón está en que puede superar todas las limitaciones y lograr la unidad con el objeto. La "Fenomenología" de la Enciclopedia describe justamente este proceso, de una conciencia que se va apropiando de su objeto y de su mundo. Se trata en principio de una conciencia enfrentada a una realidad presuntamente externa que poco a poco va incorporando a sí misma. Cuando tal unidad se produce podemos hablar de un logro de la razón; es la razón la que es capaz de reunir al sujeto y al objeto, a la conciencia con su mundo, pero no porque el mundo quede determinado por la conciencia, pues si así fuera solo tendríamos una forma de subjetivismo, sino porque reconoce una especie de ámbito anterior que abarca a la conciencia y al mundo. Lo propio de la razón es reconocer un terreno sustancial, un ámbito en el que el individuo advierte que, lejos de estar enfrentado al mundo, como ocurre aún desde la perspectiva del entendimiento o de la pura conciencia, está ligado con su realidad.

Como ya lo afirmaba Hegel en su artículo crítico Creer y saber (2016b, pp. 301 y ss.), para la filosofía de Kant el sujeto y el objeto son elementos que aparecen espontáneamente divididos; en ella hay un dualismo que nunca llega a superarse, ni siquiera en el terreno de la razón práctica o del juicio estético. Lo que prima allí es la separación. En cambio, al final de la "Fenomenología" de la Enciclopedia o, mejor, en el tránsito entre la "Fenomenología" y la "Psicología", la razón aparece de dos formas, a saber, como inteligencia y como voluntad, con lo cual se quiere señalar que mediante ambos elementos se une el sujeto con el objeto (Hegel, 2016h, p. 228). ¿En qué consisten estas dos maneras de lograr la unificación? La manera teórica es la que realiza la razón en tanto inteligencia y la manera práctica es la unificación en tanto voluntad de la razón. También esta distinción ya está en la filosofía de Kant, solo que Hegel la retoma de manera distinta, pues se trata de mostrar a la razón teórica y a la razón práctica como actividades espirituales que logran la unificación de la consciencia con su mundo, algo que por lo demás en Kant nunca tiene lugar. Por un lado, Hegel muestra que la 
razón teórica puede llegar a encontrar una especie de racionalidad que abarca al todo, tanto al ser humano como a su mundo. La ciencia, por ejemplo, es un producto de la razón teórica que se propone alcanzar esa primera reunión del ser humano con la realidad. Ella no se enfrenta a la naturaleza como algo externo, sino que se propone poner en evidencia que lo natural está gobernado por leyes que no solo dominan al mundo físico sino también a nosotros en él, y que las podemos conocer. Así es como ganamos una primera forma de unidad con el mundo; superamos la confrontación entre el sujeto y el objeto, pues reconocemos a un mundo atravesado por una razón nomológica, por leyes universales y constantes matemáticas. La ciencia es, pues, la primera forma de unidad que logramos con la realidad; son los logros de una razón que ya no pelea con lo externo, sino que lo incluye dentro de una misma estructura racional de la que el sujeto se sabe parte.

La otra forma de apropiarnos de la realidad es mediante un sentido práctico. Se describe con ello el momento en que proyectamos en lo real nuestros fines para transformarlo y adecuarlo a ellos. Hegel designa esa forma de unificación racional con el concepto de voluntad, que viene a completar la primera forma de unidad solo teórica y abstracta: nos hacemos propia esa realidad, la atravesamos con nuestros proyectos. La razón práctica supera a la teórica en tanto allí no se trata de desentrañar o desocultar la estructura normativa que gobierna el mundo, sino que lo que se quiere es hacerlo propio, esto es, modificarlo y ajustarlo a los propósitos de la actividad humana; tal cosa significa reconducir el mundo a la voluntad. Se trata de una superación conceptual de la razón teórica o de poner en evidencia que esta es mejor vista cuando se la considera en un sentido práctico como nuestro interés de realizarnos en el mundo y hacérnoslo propio reconfigurándolo. Dicho de otra manera, el tránsito de la razón teórica a la razón práctica es un ir a un terreno más fundamental, al terreno de la praxis, pues allí vemos que la teoría científica es una forma de praxis o, en otra formulación, que bien vista la ciencia es una forma de realización de fines humanos.

En la Fenomenología de 1807 se pueden ubicar las distintas figuras que hemos venido comentando. La conceptualidad es similar en ambas obras. Las experiencias de la razón describen una conciencia convencida de que el mundo tiene la misma racionalidad que ella, y que asume que este responderá a su lenguaje, pues está constituido por el mismo espíritu. El modelo de la inteligencia es la razón teórica científica o la "razón observante" (Hegel, 2016c, pp. 185 y ss.), 
que ya no ve el mundo como algo opuesto, sino unificado mediante una estructura racional. El arquetipo de la razón práctica, por su parte, es el de aquel que quiere modificar el mundo y lo ajusta a sus propios fines (Hegel, 2016c, pp. 263 y ss.). También aquí la "razón observante" es solo teórica, y corresponde a la conciencia científica que asume que el mundo está notado por la racionalidad de la ciencia. Pero esta razón teórica no realiza una unidad plena con la realidad. La experiencia del individuo teórico es que algo de él no se ha realizado, que él es incompleto, que su unidad con la realidad no se da plenamente si el mundo humano y social no es también determinado por esa razón. En el tránsito al mundo práctico no se trata, desde luego, de observar para descubrir formas racionales, sino de realizar mediante la acción el germen racional que el individuo cree con certeza que está latente en lo social. Las experiencias de esta razón práctica son las que analiza la sección que se pregunta por una "Autoconciencia racional que se realiza a sí misma” (Hegel, 2016c, pp. 263 y ss.).

El asunto que salta a la vista aquí es que la Fenomenología del espíritu de 1807 es una exposición distinta de la presentación de la Enciclopedia, pues allí las dialécticas están iluminadas por figuras vívidas tomadas de la experiencia concreta ${ }^{1}$.

1 Podemos remontar la noción hegeliana de experiencia a Platón y Aristóteles. La experiencia en Aristóteles implica una forma de acceso a la verdad previa a la episteme o al saber fundado en el conocimiento de causas universales; no se compara, pues, en jerarquía y rigor con la episteme y su conocimiento, pero hay en ella verdad. Aristóteles compara la episteme con el conocimiento del médico, que conoce las causas verdaderas de la enfermedad, y la experiencia con el saber práctico del curandero, que está ligado a una suerte de ejercicio cotidiano y que no es desdeñable. La experiencia está entonces ligada a una práctica, es decir, es un saber no teórico, pero funcional. También la tradición de la retórica desde Platón expresa esta idea de experiencia (Platón, 1988). El filósofo conoce la idea y desde allí argumenta, el retórico por su parte no conoce la idea de la verdad, pero tiene un conocimiento de gentes, del público, de cómo hablar a la multitud para persuadirla. Sabe, en consecuencia, dirigirse a la gente para construir discursos que convencen, aun cuando sea inferior al filósofo. Hegel rehabilita estas tradiciones de manera central. Por eso en cada figura de la conciencia que presenta la Fenomenología se debe entender que se refiere a una manera no teórica, sino práctica de captar el mundo. Se trata de experiencias ligadas a usos establecidos y regidos por cierta normatividad. Para una introducción al concepto de experiencia en la historia de la filosofía, y en especial sobre el papel de la noción de experiencia en la filosofía de Kant, véase Gutiérrez, 2008, pp. 145 y ss. Fue Gadamer quien se apropió del concepto hegeliano de experiencia al señalar que la experiencia describe el movimiento de las tradiciones. Para él, experiencia es el nombre para aquellas situaciones, propias del movimiento vital de toda tradición, en las que un evento inédito o una reconfiguración de las fuerzas de una época quebrantan la continuidad del sentido, de modo que nos enfrentamos a rupturas en la comprensión y a la tarea de reformular las referencias de 
Allí Hegel apela a fenómenos concretos para demostrar que una apropiación teórica del mundo no basta para sentirnos compenetrados con la realidad o, en otra formulación, que la pura ciencia no es suficiente para hacernos conscientes de nuestras resonancias con el mundo. ¿Cuál es, por ejemplo, la figura que reúne al hombre teórico con el hombre práctico? ¿Qué experiencia concreta pone en evidencia la insuficiencia de una razón solo teórica y muestra la necesidad de transitar a un mundo práctico? La figura que usa Hegel (2016c, pp. 270 y ss.) es el Fausto de Goethe. ¿Qué le sucede a Fausto? Él es el modelo del individuo teórico, del sabelotodo, por así decir, pero lo que le sucede es que se enamora. Este sabio tiene todo el conocimiento teórico del mundo, pero es incapaz de conseguir la atención y el favor de una joven llamada Margarita. Se da cuenta, entonces, de que a su experiencia de mundo le falta algo, que carece de una comunión más directa con la realidad que no se logra ya mediante la razón teórica, sino a través de una inmersión más profunda en el mundo. Lo que pone en evidencia la figura de Fausto es que nuestra simple aprehensión teórica de la realidad puede hacernos despertar el sentimiento de unidad con ella, pero que tal sentimiento no basta. Necesitamos sentir que en realidad el mundo se ajusta de verdad a nuestros fines y deseos. Después de la figura de Fausto aparecen por eso modelos de experiencias concretas que ponen en evidencia esa pretensión de la unidad del ser humano con su mundo. El significado conceptual de la figura de Fausto es entonces el siguiente: como él no es un individuo activo capaz de realizarse en su medio social, entonces fracasa en su intento por lograr una unidad con el mundo; es como si él y su medio social hablaran lenguajes diferentes. Hace falta entonces una experiencia que tome el orden social como una estructura que debe adquirir su forma racional mediante la acción humana. Con ello, transitamos a las figuras de la razón práctica.

La Fenomenología del espiritu ofrece más adelante varios protagonistas de la razón práctica. Así, una de las figuras a las que acude es la del revolucionario, aquel individuo que se propone hacer que el mundo y la realidad funcionen de

significación, a fin de restaurar la continuidad del espacio agrietado. La experiencia describe el momento en que la inercia de lo sobreentendido se rompe (Gadamer, 1960, pp. 334 y ss.) y allí está la clave de la dialéctica (Gadamer, 1971, p. 99). Sobre la tensa relación entre los conceptos de experiencia en Hegel y Gadamer, véase Gama, 2002, p. 41 y ss., y Forero, 2017, pp. 43 y ss. 
acuerdo con una razón que, aunque considera inscrita en las cosas, asume también que requiere de su acción para hacerse real y efectiva (Hegel, 2016c, pp. 275 y ss.). El revolucionario no toma la realidad como un elemento extrínseco, sino reconoce que está regida por una racionalidad que debe despertarse mediante la acción. La praxis revolucionaria es la realización de una racionalidad latente, es decir, es la plasmación concreta de lo racional inserto en el mundo que el revolucionario actualiza con su actividad. Este personaje pretende entonces activar una racionalidad que habita en el mundo, de modo que la realidad no se le aparece como pura nulidad o externalidad. El desarrollo ulterior de esta dialéctica muestra la decepción del revolucionario; él cree que el mundo se va a ajustar bellamente a su pretensión, pero encontrará que lo real le presenta obstáculos insalvables. Más aún, al proponerse realizar su ideal de bien, termina implicándose en resultados que no había previsto, porque al actuar su acción ya no hace pa rte de él, de suerte que termina generando consecuencias insospechadas. Desea, por ejemplo, imponer un modelo de justicia y ello deriva en la tiranía más absurda; con la mejor intención y con la presunta certeza y conciencia de una racionalidad inscrita en el mundo, termina desfigurando su ideal del bien. En últimas, lo que le enseña la experiencia al revolucionario es que el orden social se le resiste. Él es un individuo que aún trata de imponerse sobre la realidad social en la que habita, de suerte que solo la combate para lograr su realización.

Pues bien, ¿qué es lo que se anuncia en estas figuras de la Fenomenología del espiritu? En esta obra se combinan de forma no siempre evidente planteamientos altamente especulativos con análisis de fenómenos literarios, sociales y culturales que son familiares para todos. Se trata del permanente entrelazamiento entre planteamientos que remiten a la estructura puramente conceptual del desarrollo filosófico que tiene lugar en la obra y las alusiones a figuras literarias, históricas o mitológicas que encarnan experiencias constitutivas de todo ser humano y de toda comunidad. Podríamos también nombrar algunas figuras más, como las del reconocimiento, la familia y la vida en comunidad, la búsqueda de la trascendencia mediante el arte o la religión, entre otras. La Fenomenología trenza enunciados del más elevado tecnicismo con frases hondamente emocionales que cautivan al lector y le hacen reconocer las implicaciones existenciales de lo que allí se expone. En últimas, la obra presenta la recíproca influencia y condicionalidad que vincula el ámbito del movimiento especulativo con el acontecer real, 
la exposición del absoluto con la experiencia histórica, el puro pensar filosófico y la situación efectiva de los individuos. En efecto, lo que vemos mediante las figuras del Fausto y el revolucionario es que al interior de las diferentes estaciones que expone la Fenomenología, por las cuales se va manifestando el absoluto, se debe mostrar la experiencia concreta o la forma de vida particular que lleva a una conciencia natural a sostener una verdad; se deben poner en evidencia las creencias con las que la conciencia organiza alrededor de sí toda una forma de vida, así como aquellas otras experiencias que hacen que esta visión de mundo resulte insostenible y que sea a la postre sustituida por otra nueva. En tanto que a la conciencia se la va guiando en la Fenomenología por estas experiencias, es ella misma la que al final se convence de la presencia cada vez más clara del absoluto en su praxis total.

Es cierto que con frecuencia el mismo Hegel contribuye a que se pierda de vista la relación entre filosofía y experiencia, pues no solo en sus referencias a la Fenomenología en sus obras posteriores silencia este nexo, sino que incluso en el desarrollo mismo del texto de 1807 no siempre hace visible el hilo de la experiencia que debería articular el desarrollo filosófico. La obra se presenta en la "Introducción" como la exposición completa del camino de la experiencia de la conciencia natural gobernada por una dialéctica negativa, pero parece que cuanto más avanza el texto tanto más difícil es identificar la dialéctica de la experiencia que, según Hegel (2016c, p. 80), gobierna el tránsito entre las distintas figuras de la conciencia analizadas. Sin embargo, una mirada atenta es capaz de reconocer que en toda la Fenomenología es visible su enlace con la experiencia, incluso en las últimas secciones del libro, donde se puede ver que la obra nunca corta definitivamente todos sus enlaces con el ámbito de la experiencia humana, ni siquiera en las mayores alturas de la especulación ${ }^{2}$.

2 En este sentido, las investigaciones recientes han mostrado que se puede hacer valer hasta el final de la Fenomenología del espiritu la presencia de los dos personajes con los que se trama la obra: la conciencia natural que hace experiencia y el fenomenólogo que la conceptualiza (Bouton, 2010, pp. 107 y ss.). Es posible mostrar que las verdades que elabora la conciencia natural, desde la figura de la buena conciencia hasta el saber absoluto, están gobernadas por la misma lógica descrita en la introducción a la obra: la conciencia fija un objeto y, al hacer experiencia, el mundo se le termina revelando de tal manera que la obliga a asumir una nueva postura. Se trata de hacer valer el hecho de que también estas figuras tienen un correlato en la experiencia, abren o recomponen visiones 
Pues bien, ya con esto estamos en condiciones de atacar la profunda diferencia que a nuestro juicio se plantea entre la "Fenomenología" de la Enciclopedia y la Fenomenología del espiritu de 1807.

2. Filosofía y experiencia. El rasgo de la Fenomenología del espiritu de 1807

CuAndo en 1831, poco antes de su muerte, Hegel emprende una revisión de la Fenomenología del espiritu con miras a una nueva edición, afirma en una nota a la obra que se trata de un trabajo de juventud "peculiar" y que no hace falta "reelaborarlo" (Siep, 2000, p. 23). Hegel habla allí de la Fenomenología como de un trabajo de juventud y esto ya nos da una indicación sobre su lugar en el sistema posterior. Pues lo que anima la filosofía de Hegel en sus comienzos son las experiencias históricas de la época o, en otras palabras, aquellas realidades contemporáneas que él había recogido y elaborado en Tubinga, Berna, Frankfurt y que se había llevado consigo a Jena. Para el joven profesor Hegel, como lo señala la introducción al Escrito sobre la diferencia entre los sistemas de Fichte y Schelling, la "antesala de la filosofía" o el "presupuesto de la razón" son las experiencias de la época o la vida inmediata de los hombres de su tiempo (Hegel, 2016b, p. 24). Él está convencido de que la época debe ser elaborada conceptualmente por la filosofía. La cuestión de cuál es la época en que vivimos la percibe, entonces, como un problema fundamental del pensamiento. Como sabemos, por este camino Hegel llega a advertir que el rasgo último de su tiempo es la instauración de dualidades en la experiencia humana y su escisión en esferas antitéticas. Buena parte de sus escritos tempranos ponen en evidencia los elementos de una época desgarrada. El asunto es que, para este joven profesor, el

de mundo, de suerte que no son exposiciones filosóficas articuladas en su pura necesidad conceptual, sino que la actividad del filósofo sigue siendo la de conectar las figuras de la experiencia de la conciencia como el hilo que despliega la razón. Por eso, para estas lecturas recientes en la figura del saber absoluto la conciencia inmersa en la experiencia no tiene idea del lenguaje conceptual de lo absoluto, pero lo integra en su forma de acceso a la realidad y en sus prácticas. El filósofo, por su parte, reconoce allí el despliegue de un trasfondo racional último. En este sentido, se puede sostener que la figura del saber absoluto revela la temporalidad (Gama, 2007, pp. 33 y ss.). Acerca de los dos personajes que protagonizan la obra, véase Flach, 2012, p. 23. 
pensamiento arraiga en la historia, en la realidad concreta; la filosofía surge de una experiencia vital e inquietante con la realidad de su tiempo (Hegel, 2016b, p. 20). La filosofía no puede situarse en una lucha a brazo partido contra otras filosofías o en la altiplanicie de la tradición espiritual, sino que emerge de un diálogo con su tiempo. Es por ello que en el mundo moderno la experiencia del pensar se convierte en pura reflexión y que sus resultados no hacen sino expresar y sistematizar los conflictos y tensiones de la sociedad. Ahora, a la inversa, el Hegel de entonces también está convencido de que, contra las positividades de una época desgarrada, hay que movilizar a la filosofía como poder unificador. En tanto el rendimiento supremo de la filosofía consiste en reconocer una racionalidad presente en todas las dimensiones de la realidad, y en tanto esta razón, lejos del proceder de la reflexión y el entendimiento que diferencian y fragmentan la experiencia, muestra la unidad del sistema de la vida, la filosofía es la llamada a enfrentar el desafío de la ruptura de la experiencia. La filosofía es la aspiración a una identidad no forzada o, dicho de otra forma, su máximo rendimiento consiste en describir la unidad racional de la realidad y de la experiencia humana. La elaboración de una obra filosófica no constituye entonces la meta en sí misma del pensamiento del Hegel de Jena, sino que de lo que se trata es de encontrar en la filosofía la superación de las fracturas presentes en la vida humana.

Ahora, es en principio interesante que un Hegel sexagenario hable de la Fenomenología del espiritu como de un trabajo de juventud. Pues un rasgo presente en sus primeros trabajos filosóficos lo constituye la idea de que entre la filosofía y la experiencia de la época hay una suerte de copertenencia o correspondencia, en el sentido de que cada una responde a la otra. Al respecto ya habla el audaz texto de 1797 titulado "El más antiguo programa de un sistema del idealismo alemán", escrito junto con Hölderlin y Schelling. Allíse plantea una influencia recíproca y una mutua condicionalidad que vincula el ámbito de la filosofía con el acontecer real, el pensar filosófico y el ser de la existencia efectiva (Hegel 2016a, pp. 234-235). En sus escritos juveniles y de Jena, Hegel señala que la filosofía se hace presente en la experiencia de la época, pero porque la época misma la empuja a que se manifieste como tal o cual filosofía, y, al revés, la experiencia de la época pone en evidencia una articulación conceptual que la filosofía elabora. Pues bien, este nexo entre filosofía y experiencia se desarrolla sistemáticamente en la Fenomenología 
del espiritu y allí radica una de las "peculiaridades" de la obra de 1807, que la muestra en su singularidad con respecto a sus proyectos filosóficos posteriores. El filósofo no se queda en las alturas del concepto, sino que retorna a la situación, pero dotado de unos puntos de vista conceptuales que le permiten aclarar la experiencia concreta. La filosofía surge de la experiencia para volver a ella, desde luego no con la perspectiva desde la que partió, sino provista de una conceptualidad que le permite aclarar la situación espiritual. En el fondo, la filosofía y la experiencia tienen en los textos de juventud y de Jena un sentido circular: partimos de la experiencia para regresar a ella dotados de una conceptualidad que nos permite indagarla en su ser y es desde tal situación concreta que regresamos nuevamente al ámbito conceptual.

Hemos dicho que en el curso posterior al periodo de Jena Hegel se orienta decididamente hacia la elaboración del sistema, sea a su presentación global, como ocurre en la Enciclopedia, sea al desarrollo exhaustivo de algunas de sus partes, como ocurre en la Lógica, en la Filosofía del derecho o en las diferentes Lecciones que ofrece a sus estudiantes. Ahora bien, Hegel nunca le dedicó a la Fenomenología del espiritu de 1807 uno de los cursos que dictó como profesor y al parecer muy pronto dejó de considerarla como una introducción al sistema de la ciencia, es decir, rápidamente se alejó del modo como en principio presentó esta obra (de la Maza, 2017, p. 480). Sin embargo, ello no significa que la Fenomenología del espiritu fuera refutada y menos aún olvidada, si se considera el hecho de que Hegel se refiere a ella en numerosas ocasiones en sus cursos universitarios y en varias de las ampliaciones del sistema. La Fenomenología aparece ante los ojos del Hegel maduro como una obra de actualidad en sus propios términos, pero a la vez como un texto "peculiar". Tal formulación significa que esta obra no se deja incluir en el sistema, pues se trata de un texto elaborado desde una perspectiva única distinta a la que gobierna las obras posteriores a 1807.

Pero, ¿cuál es la perspectiva específica de análisis que opera en ella y que la distingue de los otros textos hegelianos posteriores, que gravitan siempre en torno a la órbita del sistema de la filosofía? La primera particularidad que salta a la vista tiene que ver con las lógicas que están presentes en la Fenomenología del espíritu de 1807 y la "Fenomenología" de la Enciclopedia. Ya al final de la "Lógica" presente en el Esbozo jenense de sistema II de 1804-1805, Hegel (1971) señaló 
la realidad de la lógica especulativa. Esta lógica mostraba cómo toda entidad, para ser determinada, remite a otra realidad; cómo los entes se relacionan internamente y terminan encadenados. Al examinar una entidad desde sí misma, ella muestra la negación que le es inherente y por esa negatividad vemos que es más compleja de lo que en principio pensábamos (Pinkard, 2000, p. 166). La realidad es especulativa o negativa. En la última sección de aquella Lógica Hegel indicaba que lo negativo nos permite compenetrarnos con el mundo. El Esbozo jenense de sistema III sobre Filosofía de la naturaleza y filosofía del espíritu de 1805-1806 se esfuerza por demostrar que en toda región de la realidad puede advertirse la efectividad de esta lógica especulativa (Hegel, 1976). La denominada Filosofía real de Jena puede ser leída entonces como un intento por reconocer esas relaciones lógicas negativas y mostrarlas en la forma en que se insertan en la realidad natural y espiritual. Se trata de poner en acción la tesis según la cual la negación atraviesa lo real: pensar especulativamente significa reconocer que ninguna entidad en el mundo físico y humano puede pensarse en aislamiento y separada del todo, sino que para ser definida debe ser engarzada en relaciones de sentido con otras entidades. La realidad en todos sus pliegues pone en evidencia, entonces, esa estructura especulativa o negativa que describe la lógica. Así es como se realiza la lógica especulativa de Jena, cuyos términos más formales fueron presentados en el Esbozo jenense de sistema II y en las reflexiones de Jena sobre la proposición especulativa, donde la realidad es vista como un proceso constante de negación y precisión del punto de partida en que nos hallamos (Forero, 2019, p. 175).

Pues bien, también la Fenomenología es la revelación de la realidad de la lógica especulativa o negativa de Jena, solo que esta vez vista desde la perspectiva de la formación de la conciencia. Si el pensar especulativo debe atender a la realidad para reconocer y describir en ella el despliegue de lo negativo, si la filosofía tiene que hacer efectivo lo especulativo en los diferentes ámbitos de la realidad, entonces la Fenomenología no es sino la demostración de que este proceso de negaciones también se hace presente en la experiencia humana. La obra muestra que el paso de una experiencia a otra significa, en el fondo, que estamos ahondando en la racionalidad de la experiencia humana. En últimas, la profunda intelección de la negatividad de la experiencia y de la filosofía que Hegel logra en la Fenomenología constituye, en sus rasgos distintivos, una prosecución de la lógica 
especulativa de Jena ${ }^{3}$. Toda experiencia queda allí integrada dentro de un proceso de negación, por el cual vamos atravesando diversas manifestaciones de la vida humana y reconociéndolas según crecientes grados de complejidad, hasta que en su etapa última podemos hacer experiencia de la racionalidad absoluta. Gracias al proceso racional de esta lógica especulativa se va complejizando y develando la estructura conceptual de la experiencia, hasta que al final comparece el saber absoluto. Lo que le da unidad a toda la Fenomenología es entonces la lógica especulativa, y allí lo absoluto aparece como la clave organizadora de la negatividad de la experiencia humana en su conjunto.

En principio, hay una peculiaridad de la Fenomenología del espiritu de 1807 con respecto a la presentación definitiva del sistema. La lógica que atraviesa a la Fenomenología es la que Hegel presentó en 1804-1805, en la cual esta se separaba de la metafísica (Hoffmann, 2015, p. 187). Hegel (1998, p. 220) se atiene allí a una división clásica: asume que la lógica es solo la introducción al pensar

3 En efecto, junto con el lugar que ocupa la Fenomenología del espiritu al interior del sistema, otra de las cuestiones más espinosas que han acompañado la recepción de la obra es la que concierne a la lógica presente en ella. Por lo menos tres perspectivas se abren al respecto. La primera afirma que la obra tiene su propia lógica, reconocible en la frase especulativa que desarrolla el prólogo y que está presente en todo el movimiento del texto, solo que al modo de un saber filosófico imperfecto y contaminado. Esta lógica se revela al final como "lógica o filosofía especulativa" (Hegel, 2016c, p. 39). Tal lectura sostiene que la Fenomenología puede ser interpretada como una obra autónoma con respecto a los demás proyectos de sistema. Al respecto, en su ya clásico comentario sobre $L a$ lógica de la Fenomenología del espiritu, Johannes Heinrichs (1974, p. 41) señaló que la lógica especulativa está presupuesta en la obra como una conceptualidad que ya se revela en la introducción al libro. Sobre la presencia en la Fenomenología de la lógica que revela la proposición según la cual la sustancia es a la vez sujeto, véase Pippin, 2008, pp. 19-20, y acerca de la proposición especulativa en aquella obra, Zeidler, 2012, pp. 142 y ss. La segunda posición asegura que la lógica que opera en la Fenomenología fue proyectada en el Esbozo jenense de sistema II de 1804-1805. Esta obra culmina en la exposición de la estructura conceptual de la realidad, donde Hegel identifica el conocer que reúne dos premisas presuntamente antagónicas (las de la unidad inmediata y la diferencia) y una tercera premisa, que restaura la unidad de lo contrapuesto. Esta segunda lectura ofrece la ventaja de ver la génesis de la Fenomenología en los trabajos de Jena. A mi modo de ver, esta tesis es absolutamente verosímil: la Fenomenología es resultado de un desarrollo filosófico que empieza a perfilarse desde Jena e incluso desde los escritos de juventud (Forero, 2019, pp. 211 y ss.). La última posición es la que trata de vincular la Fenomenología del espíritu de 1807 con la Ciencia de la lógica de 1812 diciendo que aquella obra es solo una introducción o puerta de acceso al sistema de la ciencia tal y como Hegel lo había planeado entre 1807 y 1812 (Claesges, 1981, p. 134). Sobre este asunto, véase Aschenberg, 2012, pp. 88 y ss. Para una introducción general a la estructura de la Fenomenología del espiritu leída desde sí misma, véase Díaz, 1986, p. 5. 
especulativo, es decir, que su tarea es solo superar las formas de pensamiento finito. Esta lógica se propone, pues, transitar desde las presentaciones del pensamiento reflexivo y del entendimiento a un pensar propiamente especulativo. La lógica de 1804-1805 es, en consecuencia, el estudio o doctrina del pensamiento, pero aún hace falta ocuparse del ser de las cosas, es decir, de la metafísica. Allí la lógica tiene que pasar al análisis de los fenómenos inmediatos, de modo que solo se realiza como metafísica, filosofía de la naturaleza y fenomenología del espíritu. En cambio, la lógica de 1812-1816, presente en la Enciclopedia, asume que las formas finitas del pensar son momentos dentro del despliegue de la razón en su totalidad (Hegel, 2016d, p. 187). Como la Ciencia de la lógica describe de inmediato la estructura última de la realidad, se rompe la división clásica entre lógica y metafísica; la lógica y la metafísica son lo mismo (Hegel, 2016e, p. 65). Lo peculiar de la Fenomenología de 1807 radica en que esta obra está atravesada por una lógica que solo introduce al pensar, sin incluir en ella la exposición del ser mismo, que tiene lugar cuando se va a los fenómenos concretos. La Lógica de 1812-1816, por su parte, es ya el desarrollo de la pregunta por el ser mismo de la realidad, y, si bien está presente en la "Fenomenología" de la Enciclopedia, no es allí donde se desarrolla como ciencia básica, pues su exposición principal ya tuvo lugar en la primera parte del sistema como metafísica.

Más aún, la Fenomenología del espiritu de 1807 se propone, en principio, revelar el mismo resultado que presentó la lógica de 1804-1805, a saber, superar la brecha entre pensar y ser, y revelar una estructura conceptual que es la sangre misma de la realidad. Por eso la dirección de ambas obras se traslapa en algún punto. En últimas, se trata de apuntar al mismo núcleo de la racionalidad universal, solo que una vez desde la perspectiva del análisis conceptual (lógica de 1804-1805) y otra vez desde la formación de una conciencia natural a la que la experiencia le va revelando la racionalidad en la que habita (Fenomenología del espiritu). En ambas formulaciones se despliega la misma racionalidad, pero alumbrada con reflectores diferentes. La idea de Hegel es, en el fondo, que la misma racionalidad circula en las diferentes presentaciones del pensar, una vez como lógica de 1804-1805 y otra vez como Fenomenología del espiritu (Becker, 1971, p. 138).

Lo peculiar y específico de la Fenomenología de 1807 reside más decididamente, sin embargo, en la conexión con la experiencia que ya señalamos, es decir, 
en el hecho de que allí todos los planteamientos presentados, incluso los más especulativos, no son solo proposiciones que hacen parte de un sistema científico, cuya verdad se deduce y asegura en su nexo lógico y filosófico con otras proposiciones, sino enunciados cuya verdad requiere de una realización en la experiencia. No es que la Fenomenología haga de la experiencia, como en el empirismo, la base absoluta de la que procede toda verdad, ni de un empirismo craso que se mantenga en el nivel más inmediato de la experiencia, y tampoco se trata de volver a la experiencia, como en el racionalismo, solo para confirmar en ella conocimientos ya garantizados y asegurados por la pura razón. Tampoco es cuestión, como en Kant, de partir de la experiencia para poner en evidencia las condiciones trascendentales que la hacen posible, pero que ya no harían parte de ella. Para Hegel, y esto es lo que se hace evidente únicamente en la Fenomenología y que ya aparecía en los textos de Jena, la esfera de la experiencia se engrana con la esfera conceptual sistemáticamente organizada, de forma tal que ninguna es base y fundamento de la otra, sino que solo en su mutuo entrelazamiento se alcanza una auténtica elaboración. En su dependencia del concepto, la experiencia adquiere una hondura dialéctica ausente en la concepción corriente que reduce la experiencia a la simple percepción sensible, mientras que en su dependencia de la experiencia, las verdades conceptuales adquieren una vitalidad y existencia efectiva ausentes tanto en la concepción abstracta de ser del empirismo y del racionalismo, como en la filosofía trascendental de Kant. Entre el concepto y la experiencia, entre la filosofía y la vida, no hay una región que ocupe el lugar de fundamento de la otra, como tampoco hay aquí un plano trascendental que no haga parte de la experiencia; más bien, solo hay experiencia real si ella está determinada desde el nivel del concepto, pero la filosofía solo es verdadera si se muestra su efectividad en el orden concreto de la experiencia.

La peculiaridad de la Fenomenología del espiritu y de varios trabajos de juventud y de Jena de Hegel reside, así, en esta permanente remisión al ámbito de la experiencia, no como a la esfera donde se corroboran verdades conceptuales ya aseguradas de antemano, sino ante todo como ámbito donde dichas verdades se hacen efectivas y ganan realidad. El horizonte de la experiencia y el del concepto se vinculan como dos elementos que se refieren recíprocamente. De allí el carácter de movilidad permanente que acompaña toda la exposición de la Fenomenología y que puede molestar al lector habituado a textos filosóficos que 
presentan una serie de tesis sostenidas por encadenamientos puramente conceptuales. El conjunto de proposiciones que se analizan en la obra no representa entonces meras verdades enunciadas que, en su engranaje sistemático, describieran la esencia última de las cosas; en cambio, ellas son ante todo verdades que se encarnan en la praxis humana misma, reflejando la posición de un individuo o de una comunidad ante la existencia y el mundo. La oscilación constante que en la Fenomenología va de la experiencia a la ciencia, y luego desde la filosofía al ámbito de la experiencia concreta, le confiere un talante de movilidad y transitoriedad constantes. Allí todo enunciado que brota de la observación fenomenológica de la experiencia se remite de inmediato al trasfondo conceptual que en él opera, pero de nuevo esa determinación conceptual se hace retornar al horizonte de la experiencia real, porque solo allí gana existencia y sentido.

Podemos reconocer este rasgo de la Fenomenología mediante una circunstancia a primera vista de interés solo anecdótico. Sabemos que antes de decidir su título definitivo, Hegel había considerado titular esta obra con el nombre de Ciencia de la experiencia de la conciencia y parece que este es el título con el que comenzó a imprimirse el libro. En enero de 1807 Hegel añade a la extensa obra un prólogo voluminoso y es entonces cuando decide cambiar su nombre por el definitivo de Fenomenología del espiritu. No nos interesa indagar por las razones históricas de esta decisión; más bien, importa destacar que los dos títulos reflejan por igual el propósito y el sentido de la obra, a saber, el de entroncar la filosofía con la experiencia, pues las verdades conceptuales solo tienen sentido si se abren y se asientan en el mundo real sobre el que los seres humanos despliegan sus prácticas y su vida concreta. El título Fenomenología del espiritu describe un análisis de las distintas manifestaciones del espíritu que conduciría al desarrollo último de la filosofía o, en otras palabras, señala el propósito de elaborar las figuras en que se despliega la vida humana como parte de una ciencia filosófica. Cuando Hegel quiere acentuar el hecho de que lo racional se inserta en la realidad humana, lo llama espíritu. Este término tiene desde luego un contenido teológico fuerte. En la doctrina cristiana, el espíritu es la mediación entre el padre trascendente y el hijo que está en la tierra, de manera que describe la fuerza que permite que el ser no quede alejado de la realidad, sino que se inserte en la vida de los hombres y se haga real. Pues bien, este proyecto de trenzar la filosofía con la experiencia que resuena en el nombre Fenomenología del espiritu puede también 
reconocerse en el intento por realizar un análisis científico de la experiencia de la conciencia o, como reza el otro título de la obra, como una Ciencia de la experiencia de la conciencia. En efecto, es en la medida en que cada verdad ligada a la experiencia es traducida en la Fenomenología, según su más profundo significado conceptual, que esta obra puede ser descrita como una Ciencia de la experiencia de la conciencia.

Más allá de la circunstancia histórica de la vacilación de Hegel entre dos títulos, lo que ponen en evidencia ambos nombres es que allí se conjugan dos procesos que se complementan y condicionan mutuamente. La paulatina manifestación del espíritu que va articulando el sistema solo es posible desde una consideración científica de la experiencia y, a la inversa, tal examen científico solo tiene lugar si cada experiencia se integra en una totalidad sistemática, en lugar de ser analizada separadamente. El libro es una elaboración filosófica desde la experiencia humana concreta, pero también se puede decir que, al revés, muestra que dicha experiencia no consiste en una amalgama o superposición de realizaciones vitales de la más diversa índole e imposibles de vincular entre sí, sino que es posible articular las distintas formas de su realización en una exposición científica. Por ello es una Ciencia de la experiencia de la conciencia ${ }^{4}$. Ambos títulos señalan la permanente oscilación que lleva a la obra de la experiencia a la ciencia y luego

4 Desde luego, defender esta unidad de los títulos de la obra es ya asumir la unidad del texto. Algunos comentaristas afirman que la Fenomenología del espíritu como Ciencia de la experiencia de la conciencia se extiende desde las figuras de la "Conciencia" hasta las de la "Razón" y que para entender esas secciones hay que tener presente la introducción a la obra. El título Fenomenología del espiritu, por su parte, describiría lo que se elabora entre las figuras del "Espíritu” y el "Saber absoluto". Se trataría en este segundo caso casi de un nuevo proyecto en el cual Hegel, en últimas, estaría perfilando la Ciencia de la lógica. Según estos comentarios, para comprender estas secciones con las que cierra la obra, así como su ligazón con la Lógica y con el sistema posterior, sería clave, no ya la introducción a la Fenomenología, sino su prólogo. Se trataría, en conclusión, de dos proyectos que habitan en la misma obra, anunciados por los dos títulos con los que fue concebida y por la introducción y el prólogo con los que abre. En este sentido, desde Rudolf Hyam (1857), en el siglo XIX , y Theodor Haering, en el siglo xx, se ha debatido la cuestión de si hubo, durante la redacción de la Fenomenologia, un cambio de proyecto. Al respecto, el dictamen de Theodor Haering (1934) es que esta obra "no surgió en Hegel de manera orgánica y al hilo de un plan cuidadosa y largamente meditado y trazado, ni de su desarrollo precedente, sino como consecuencia de una decisión repentina” (p. 193). Lo que acabamos de señalar sobre los títulos indica, al contrario, que se trata de dos maneras de nombrar el mismo proyecto unitario. Para una introducción a este asunto, véase Bonsiepen, 1977, pp. 59-60, y Pöggeler, 1973, pp. 191 y ss. 
desde la filosofía al ser de lo real, su condición de doble puerta de despliegue de la filosofía y de manifestación de la experiencia humana.

Pues bien, la relación que allí se traba entre filosofía y experiencia también nos permite ubicar esta obra en su especificidad. En otras palabras, la complejidad de este texto que, como sus títulos lo indican, es dos cosas a la vez -conceptualidad de la experiencia y realización concreta del concepto o espíritu-, le confiere un lugar particular. Lo específico de esta obra es la excepcional integración entre experiencia y concepto, o entre vida y filosofía. La filosofía concebida como ciencia guarda allí un nexo íntimo con la experiencia, es decir, hay una conexión entre el concepto y la praxis concreta de los individuos y las culturas. Dicho de otra forma, en la Fenomenología se trata de que la filosofía busque una conexión con la vida o con la experiencia más inmediata, con el activo y práctico estar en el mundo de la conciencia. No es que esta obra sea sustituida en el sistema posterior por una elaboración más sistemática de la filosofía, que es lo que se hace predominante después de 1807. En la Fenomenología, la filosofía solo se realiza desde una consideración de la experiencia. Podemos decir entonces que la Fenomenología está fuera del sistema posterior o, por lo menos, que puede ser leída en sí misma, en su valor propio, y no como preparación o introducción o como una parte del sistema ulterior, ni como un planteamiento que después fuera refutado por este, ni como la elaboración de una sección que haría parte de la "Filosofía del espíritu" presentada en la Enciclopedia de las ciencias filosóficas.

Nuestro planteamiento se distancia, entonces, de las dos lecturas más conocidas sobre la posición de la Fenomenología al interior del sistema. La Fenomenología no es la introducción al sistema de la ciencia tal y como Hegel lo planeó en 1807, y por tanto a esta obra no sigue la Ciencia de la lógica, pero la obra tampoco es la misma que se presenta como "Fenomenología del espíritu" en la exposición definitiva del sistema. A nuestro juicio, se trata de una obra autónoma con respecto al proyecto del sistema de la ciencia posterior. Como en la $\mathrm{Fe}$ nomenología del espiritu de 1807 la experiencia humana juega un papel decisivo como vía de acceso al saber filosófico, esto es, al conocimiento del concepto, a la ciencia y por ello a la estructura última de lo real; como el nexo de la filosofía con la experiencia resulta ser un elemento esencial y constitutivo, entonces esta es una obra particular que no se puede ubicar dentro del sistema último de Hegel. 
La experiencia da allí lugar al desarrollo filosófico, de modo que, lejos de ser un tema menor en la obra de 1807, es el elemento central y único de este trabajo.

No obstante, habría que preguntar si la relación entre filosofía y experiencia no se encauza en otra dirección o se transforma en el Hegel posterior. Pues podría ser que la Fenomenología no sea la única obra en la que la filosofía tenga una vocación hacia la experiencia, y que las alusiones a la experiencia del sistema posterior no solo se traigan para ilustrar una determinación de orden lógico y conceptual que en su necesidad científica debería ya bastarse a sí misma. Para terminar, deseo dar un par de señales sobre este asunto.

El trabajo de Walter Kaufmann (1979, pp. 107 y ss.) reveló las circunstancias personales que atravesaba Hegel en el momento en que concluía la Fenomenología. Los biógrafos han contado, además, que las últimas páginas de la obra fueron escritas en la noche del 12 al 13 de octubre de 1806, en el mismo instante en que se escuchaban los cañones de Napoleón, que en la batalla de Jena puso fin al Imperio Romano Germánico. El libro se terminaba el mismo día en que un acontecimiento puntual encauzaba la historia europea hacia un nuevo mundo, a saber, hacia la realización de los ideales ilustrados que animaron la revolución (Hyppolite, 1968, p. 9). Este nuevo producto del pensamiento coincidía pues con la apertura de una nueva experiencia y de una nueva época. La terminación del libro confirmaba así un planteamiento central de la obra de Hegel: la condicionalidad mutua entre lo especulativo y el devenir concreto, entre el concepto y la experiencia histórica, entre el pensar filosófico y la vida efectiva (Hyppolite, 1968, p. 33). ¿Desaparece este lazo de la obra de Hegel? En algún sentido, lo que Hegel desarrolla en el "Espíritu objetivo" de la Enciclopedia va en la misma dirección que la Fenomenología del espiritu. El propósito último del "Espíritu objetivo" es ayudar a hacer efectiva una nueva verdad y con ello un nuevo mundo espiritual o, en otras palabras, allí también se trata de un nacimiento conjunto entre un pensar y una nueva época. Aquella sección presenta un pensar universal que prepara un nuevo momento espiritual y que trata de dotar a la realidad de sus principios; se trata, otra vez, de hacer confluir la singularidad del pensamiento universal con la gestación de un periodo histórico, y que el individuo que se aproxime a aquella obra gane conciencia de su lugar en el nuevo mundo y en la existencia.

Por otro lado, ocasionalmente Hegel distingue en su sistema un punto de vista filosófico de uno no filosófico, tal y como lo hace en la Fenomenología de 1807. 
Sabemos que en la Fenomenología el "para nosotros" tiene un papel determinante, es el filósofo que reúne las figuras de la experiencia, y se contrapone a la conciencia que hace la experiencia. Ahora bien, en la Ciencia de la lógica Hegel habla de un "para nosotros", que es el filósofo que lleva todo el desarrollo de las determinaciones del pensamiento, y en el parágrafo 381 de la Enciclopedia también señala un "para nosotros". En este parágrafo de la Enciclopedia el "para nosotros" describe al filósofo que sistematiza la naturaleza, que aparece como un presupuesto del espíritu, y esa posición se contrapone a la realidad de la experiencia, en la cual ese presupuesto no se da. Hegel parece indicar que el sistema es "para nosotros", quienes hacemos filosofía, y queremos darle una secuencia a la realidad, pero no más; la realidad de la experiencia podría tomar cauces inesperados que la filosofía misma debería reintegrar en el sistema. La Enciclopedia se revela entonces como una construcción sistemática de todos los saberes filosóficos (lógica, filosofía de la naturaleza y filosofía del espíritu), pero elaborada desde el punto de vista de la filosofía que quiere articular lo ente, y señala a la vez que la realidad de la experiencia puede tomar una dirección distinta. Desde luego, estos nuevos rumbos deben conducir a una reelaboración del sistema, de modo que este ha de estar atento al acontecer real. La Enciclopedia no aparece pues como una obra depurada de todo contenido experiencial e histórico concreto, sino que allí se prosigue el proyecto por el cual la filosofía se realiza desde la atención a la experiencia; por lo menos, no se trata de una obra desligada de los aspectos más ligados con la experiencia humana. El desarrollo de estos asuntos sobrepasa, sin embargo, el interés de este artículo.

Referencias

Aschenberg, R. (2012). Das Recht des Bewußtseins. Eine These der Phänomenologie des Geistes und ihre System -und Kritik-Funktion. En: M. Gerten (Ed.), Hegel und die Phänomenologie des Geistes. Neue Perspektiven und Interpretrationsansätze (pp. 83-105). Würzburg: Königshausen \& Neumann.

Becker, W. (1971). Hegels Phänomenologie des Geistes. Eine Interpretation. Stuttgart: Kohlhammer.

Bonsiepen, W. (1977). "Phänomenologie des Geistes". En: O. Pöggeler (Ed.), Hegel (pp. 59-74). Freiburg: Verlag Karl Alber. 
Bouton, C. (2010). Die helle Nacht des Nichts. Zeit und Negativität bei Hegel und Heidegger. En: W. Jaeschke \& L. Siep (Eds.), Hegel-Studien (Vol. 45) (pp. 103-121). Stuttgart: Felix Meiner.

Claesges, I. (1981). Darstellung des ercheinenden Wissens. En: F. Nicolin \& O. Pöggeler (Eds.), Hegel-Studien [Suplemento 21] (pp. 81-102). Bonn: Bouvier Verlag.

Díaz, J. A. (1986). La estructura de la Fenomenología del espiritu. Ideas y Valores, 36(70), 3-32.

Flach, W. (2012). Begreifen und Begründen. Zur Einschätzung der Phänomenologie des Geistes. En: M. Gerten (Ed.), Hegel und die Phänomenologie des Geistes. Neue Perspektiven und Interpretrationsansätze (pp. 21-29). Würzburg: Königshausen \& Neumann.

Forero, F. (2017). Ontología del acontecer. El camino de Hans-Georg Gadamer. Ideas y Valores, 66(164), 35-54. DOI: 10.15446/ideasyvalores. v66n 164.55658

Forero, F. (2019). Filosofia y negatividad. Sobre el camino del pensar de Hegel en Jena. Bogotá: Universidad Nacional de Colombia.

Gadamer, H-G. (1960). Wahrheit und Methode. Grundzüge einer philosophischen Hermeneutik. Tübingen: J. C. B. Mohr (Paul Siebeck).

Gadamer, H-G. (1971). Hegels Dialektik. Sechs hermeneutische Studien. Tübingen: Paul Siebeck.

Gama, L. E. (2002). Una tensa cercanía. Gadamer y el concepto hegeliano de experiencia. Ideas y Valores, 51(120), 41-78.

Gama, L. E. (2007). Historia, olvido y recuerdo en Hegel y Nietzsche. Areté. Revista de Filosofía, 19(1), 9-39.

Gutiérrez, C. B. (2008). Ensayos hermenéuticos. México: Siglo XXI Editores.

Haering, T. (1934). Die Entstehungsgeschichte der Phänomenologie des Geistes. En: B. Wigersma (Ed.), Verhandlungen des dritten Hegelkongress in Rom (pp. 118-138). Tübingen/Haarlem: J.C.B. Mohr (P. Siebeck).

Hegel, G. W. F. (2005). Enciclopedia de las ciencias filosóficas. (Trad. Ramón Valls Plana). Madrid: Alianza Editorial.

Hegel, G. W. F. (2010). Fenomenología del espiritu. Madrid: Universidad Autónoma de Madrid. 
Hegel, G. W. F. (2016a). Werke (Vol. I). Frühe Schriften. Frankfurt am Main: Suhrkamp.

Hegel. G. W. F. (2016b). Werke (Vol. II).Jenaer Schriftlen 1801-1807. Frankfurt am Main: Suhrkamp.

Hegel, G. W. F. (2016c). Werke (Vol. III) Phänomenologie des Geistes. Frankfurt am Main: Suhrkamp.

Hegel, G. W. F. (2016d). Werke (Vol. V). Wisenschaft der Logik. Frankfurt am Main: Suhrkamp.

Hegel, G. W. F. (2016e). Werke (Vol. VI). Wisenschaft der Logik. Frankfurt am Main: Suhrkamp.

Hegel, G. W. F. (2016f). Werke (Vol. VIII). Enzyklopädie der philosophischen Wissenschaften im Grundrisse. Erster Teil. Die Wissenschaft der Logik. Frankfurt am Main: Suhrkamp.

Hegel, G. W. F. (2016g). Werke (Vol. IX). Enzyklopädie der philosophischen Wissenschaften im Grundrisse. Zweiter Teil. Die Naturphilosophie. Frankfurt am Main: Suhrkamp.

Hegel, G. W. F. (2016h). Werke (Vol. X). Enzyklopädie der philosophischen Wissenschaften im Grundrisse. Dritter Teil. Die Philosophie des Geistes. Frankfurt am Main: Suhrkamp.

Hegel, G. W. F. (1971). Gesammelte Werke (Vol. 7). Jenaer Systementwürfe II. Logik, Metaphysik und Naturphilosophie. Bonn: Felix Meiner Verlag.

Hegel, G. W. F. (1976). Gesammelte Werke (Vol. 8). Jenaer Systementwürfe III. Naturphilosophie und Philosophie des Gesites. Düsseldorf: Felix Meiner Verlag.

Hegel, G. W. F. (1998). Gesammelte Werke (Vol. 5). Schriften und Entwürfe (1799-1808). Düsseldorf: Felix Meiner Verlag.

Heinrichs, J. (1974). Die Logik der Phänomenologie des Geistes. Bonn: Bouvier Verlag.

Hoffmann, T. S. (2015). Hegel. Eine Propädeutik. Berlin: Marixverlag.

Hyam, R. (1857). Hegel und seine Zeit. Berlin: Bibliothek in Berlin.

Hyppolite, J. (1968). Genèse et structure de la Phénoménologie de l'esprit de Hegel. París: Aubier Montaigne.

Kaufmann, W. (1979). Hegel. (Trad. V. Sánchez de Zavala). Madrid: Alianza. 
de la Maza, M. (2017). La reformulación de la fenomenología del espíritu en la Enciclopedia de las ciencias filosóficas de Hegel. En: H. Neumann, O. Cubo y A. Bavaresco (Comps.), Hegel y el proyecto de una enciclopedia filosófica (pp. 473-498). Porto Alegre: RS Editores.

Pinkard, T. (2000). Hegel, a Biography. Cambridge: Cambridge University Press. Pippin, R. (2008). Eine Logik der Erfahrung? Über Hegels Phänomenologie des Geistes. En: K. Vieweg \& W. Welsch (Eds.), Hegels Phänomenologie des Geistes. Ein kooperativer Kommentar zu einem Schlüsselwerk der Moderne (pp. 13-36). Frankfurt am Main: Suhrkamp.

Platón (1988). Sofista. En: Diálogos V: Parénides, Teeteto, Sofista, Politico. (Trad. M. I. Santa Cruz, A. Vallejo Campos, N. L. Cordero). Madrid: Gredos.

Pöggeler, O. (1973). Die Komposition der Phänomenologie des Geistes. En: Materialen zur Hegels Phänomenologie des Geistes (pp. 329-390). Hamburg: Suhrkamp.

Siep, L. (2000). Der Weg der Phänomenologie des Geistes. Ein einführender Kommentar zu Hegels 'Differenzschrift' und 'Phänomenologie des Geistes'. Frankfurt am Main: Suhrkamp.

Zeidler, K. W. (2012). Zur Logik des spekulativen Satzes. En: M. Gerten (Ed.), Hegel und die Phänomenologie des Geistes. Neue Perspektiven und Interpretrationsansätze (pp. 141-149). Würzburg: Königshausen \& Neumann. 